\title{
Significant Decrease in Plasma Levels of D-Dimer, Interleukin-8, and Interleukin-12 After a 12-Month Treatment with Rosuvastatin in HIV-Infected Patients Under Antiretroviral Therapy
}

\author{
Leonardo Calza,' Vincenzo Colangeli,, Eleonora Magistrelli,, Ilaria Contadini,, Isabella Bon,? \\ Maria Carla Re, ${ }^{2}$ Matteo Conti, Rita Mancini, and Pierluigi Viale ${ }^{1}$
}

\begin{abstract}
Objectives: Statins have shown anti-inflammatory and immune-modulatory properties in both general and HIVinfected population, but their effect on plasma D-dimer levels is controversial and it has not been investigated to date in HIV-positive patients. The aim of our study was to assess the effect of rosuvastatin on D-dimer and other serum inflammation markers among these subjects.

Methods: Prospective, cohort study of HIV-1-infected adult patients receiving a stable combination antiretroviral therapy (cART), who started a lipid-lowering therapy with rosuvastatin $(10 \mathrm{mg}$ daily) and were followed up for at least 12 months. The primary endpoint was the change at month 12 in the median plasma concentration of D-dimer. The secondary endpoints included the variation in median plasma levels of these inflammatory biomarkers: interleukin-8 (IL-8), interleukin-10 (IL-10), and interleukin-12 (IL-12).

Results: Sixty-two patients were enrolled in the study, and the endpoints were available for 54 subjects. After 12 months, a significant decrease in median plasma concentration of D-dimer was observed $(-21.4 \%$; interquartile range [IQR], $-35.5 ;-4.2 ; p=.029)$. With regard to the inflammatory biomarkers, a significant decrease in median levels of IL-8 (-24.6\%; IQR, $-30.8 ;-1.8 ; p=.012)$ and IL-12 $(-18.7 \%$; IQR, $-25.8 ;+2.5 ; p=.033)$ was also observed. Rosuvastatin led to a significant reduction in serum lipid values and showed a good tolerability profile. Conclusions: Our findings show that a 12-month treatment with rosuvastatin associated with an effective cART can significantly decrease the plasma levels of D-dimer, IL-8, and IL-12, and suggest a potential role for this statin to reduce activated coagulation and systemic inflammation among HIV-infected persons.
\end{abstract}

Keywords: HIV, immune activation, antiretroviral therapy, inflammation

\section{Introduction}

C HRONIC INFLAMMATION and systemic immune activation are known to play a crucial role in the progression of HIV disease and the occurrence of noninfectious HIV-associated comorbidities, such as cardiovascular diseases. Although both inflammation and immune activation are certainly reduced by the virological suppression associated with the combination antiretroviral therapy (cART), residual inflammation and/or immune activation persist in virologically suppressed HIVinfected patients and may lead to the increased morbidity/ mortality observed in subjects with comorbidities. ${ }^{1-4}$

The identification of therapeutic approaches able to significantly reduce the residual systemic inflammation in pa- tients on cART is today a crucial challenge to define the optimal therapeutic strategy for the long-term management of the HIV disease.

The 3-hydroxy-3-methylglutaryl coenzyme A reductase inhibitors, or statins, are known to produce a potent lipidlowering effect by inhibiting the synthesis of mevalonate, the precursor of sterols. At the same time, these agents have significant anti-inflammatory and immunomodulatory properties in the general population, independent of their lipidlowering effects, and they can slow down the atherosclerotic disease progression, as demonstrated by several randomized and observational studies. ${ }^{5-8}$

Recent experimental and clinical studies have shown that statins may also act on the coagulation cascade at several

Departments of ${ }^{1}$ Medical and Surgical Sciences, Clinics of Infectious Diseases, ${ }^{2}$ Unit of Microbiology, ${ }^{3}$ Centralized Laboratory, "Alma Mater Studiorum"' University of Bologna, S. Orsola-Malpighi Hospital, Bologna, Italy. 
levels, leading to a decreased coagulation activity and thrombosis by inhibiting platelet activation, decreasing tissue plasminogen activator (tPA) and plasminogen activator inhibitor-I (PAI-I), increasing the activity of protein $\mathrm{C}$ anticoagulant pathway, and producing various effects on intracellular signaling molecules such as nuclear factor (NF)- $\kappa \mathrm{B}$ and Kruppel-like factor 2 (KLF2). However, the effect of statins on plasma D-dimer, the mostly used circulating marker of activated coagulation, is still controversial. ${ }^{9-11}$

Some clinical studies have demonstrated anti-inflammatory and immunomodulatory properties of statins in HIV-infected people too, but data are limited and not always concordant. ${ }^{12,13}$ Moreover, data are still lacking about the statin effect on the coagulation markers in HIV-infected patients.

The aim of our observational, prospective study is to evaluate the effect of a 12-month treatment with rosuvastatin on plasma levels of D-dimer, IL-8, IL-10, and IL-12 in adult HIV-infected subjects under suppressive cART.

\section{Patients and Methods}

A prospective cohort analysis of HIV-1-infected adult patients followed at our Clinic of Infectious Diseases, receiving a stable cART and starting a rosuvastatin treatment was performed. Inclusion criteria were as follows: adult patients receiving cART for at least 24 months, unchanged cART during the last 12 months, plasma HIV RNA $<20$ copies/ml for at least 6 months, current $\mathrm{CD} 4^{+} \mathrm{T}$ lymphocyte count $\geq 500$ cells $/ \mathrm{mm}^{3}$, and beginning a lipid-lowering therapy with rosuvastatin (10 mg daily) from January 1, 2013, through December 31, 2014.

Exclusion criteria were active opportunistic diseases or severe infectious diseases, acute or chronic hepatitis B virus (HBV) infection, acute or chronic hepatitis C virus (HCV) infection, acute or chronic inflammatory diseases, known cardiovascular diseases (coronary artery disease, peripheral vascular disease, or cerebrovascular disease), diabetes mellitus, alcohol or drug abuse, hypothyroidism, Cushing's syndrome, acute or chronic myopathy, acute or chronic kidney diseases, liver cirrhosis, alanine aminotransferase (ALT) or aspartate aminotransferase (AST) $>80 \mathrm{U} / \mathrm{L}$, creatinine $>1.2 \mathrm{mg} / \mathrm{dl}$, creatine phosphokinase $(\mathrm{CPK})>150 \mathrm{U} / \mathrm{L}$, pregnancy, or an underlying treatment with corticosteroids, androgens, estrogens, growth hormones, thiazide diuretics, beta-blockers, thyroid preparations, proton pump inhibitors, acid-reducing agents, statins, or other lipid-lowering drugs.

All enrolled subjects were followed up for 12 months. Patients were excluded from the final evaluation if they had any change in the current lipid-lowering treatment or in the current antiretroviral regimen during the 12-month follow-up, or if they had not a regular 12-month follow-up. The study was approved by the Ethics Committee of the S.Orsola-Malpighi Hospital in Bologna and all the enrolled patients provided a signed written consent before participating in the study.

The following demographic, clinical, and laboratory data were recorded at the start of therapy and at 3-month intervals during the 12-month follow-up: sex, age, race, body mass index (BMI), arterial pressure, clinical manifestations; serum levels of D-dimer, IL-8, IL-10, IL-12; plasma lipid levels (triglycerides, total cholesterol, LDL cholesterol, and HDL cholesterol), HIV RNA, CD4 ${ }^{+}$T lymphocyte count, glucose, complete liver and kidney function tests, CPK, aldolase, and urinalysis. All the plasma samples were analyzed for HIVRNA level using the automated COBAS AmpliPrep Instrument for specimen processing and the COBAS TaqMan Analyzer for amplification and detection (Roche Cobas AmpliPrep/Cobas TaqMan HIV-1 tests version 2.0; Roche Diagnostics, Mannheim, Germany). The limit of quantification, as defined by the manufacturer, was reduced to 20 copies/ml. Virologic failure was defined by a confirmed plasma HIV RNA $\geq 20$ copies/ml and a virological blip as a plasma HIV RNA between 20 and 200 copies/ml followed by a plasma HIV RNA $<20$ copies/ml. D-dimer, IL-8, IL-10, and IL-12 concentrations were measured by a sandwich immunological assay by an EVIDENCE analyzer (Medical Systems, Genova, Italy).

Adherence to the current therapy was carefully checked on the outpatient visits by self-reported questionnaires.

The primary endpoint was the variation between baseline and month 12 in median plasma concentration of D-dimer. The secondary endpoints included the variation during the 12-month follow-up in median plasma levels of the inflammatory biomarkers (IL-8, IL-10, and IL-12), serum lipid values (total cholesterol, LDL cholesterol, HDL cholesterol, and triglycerides), and incidence of adverse events.

The aim of the study was to evaluate whether the rosuvastatin treatment could lead to a $\geq 10 \%$ decrease in the median concentration of D-dimer and inflammatory biomarkers between baseline and month 12 . Assuming an SD of $20 \%$, a power of $80 \%$, a type I error of 0.05 , and a two-tailed Wilcoxon paired test, 40 patients needed to be enrolled in the study. Variables were described by using proportions for categorical variables, median and interquartile range (IQR) for continuous variables. To compare the variations in continuous variables between baseline and month 12 , the Wilcoxon paired test was used. All reported $p$-values are two tailed and $p$-values of $<.05$ were considered as statistically significant.

\section{Results}

Sixty-two patients were enrolled into the study and 55 subjects completed the study. Reasons for discontinuation were adverse events for four subjects (diarrhea in two cases and nausea in two cases) and virological failure with change in current cART for three subjects. All primary and secondary endpoints were available for only 54 patients who completed the study, because of missing data in one case.

Median age of the 62 enrolled patients was 46.4 years (IQR, 40.3; 55.6), 48 (77.4\%) patients were men, and 57 (91.9\%) were white. Median current $\mathrm{CD} 4^{+}$lymphocyte count was 552 cells $/ \mathrm{mm}^{3}$ (IQR, 366; 749), median duration of current cART was 35 months (IQR, 26; 47), and median duration of plasma HIV RNA <20 copies/ml was 32 months (IQR, 25; 49). Patients with total cholesterol $>200 \mathrm{mg} / \mathrm{dl}$ were $49(79 \%)$ and those with triglycerides $>200 \mathrm{mg} / \mathrm{dl}$ were 41 (66.1\%). Ongoing cART included tenofovir/emtricitabine in 37 cases (59.7\%), abacavir/lamivudine in 25 cases $(40.3 \%)$, a ritonavir-boosted protease inhibitor (PI/r) in 35 cases $(56.5 \%)$, and efavirenz in 27 cases $(43.5 \%)$.

Demographic, clinical, and laboratory characteristics of the 54 patients who completed the study and were evaluated are summarized in Table 1.

Table 2 shows changes during the 12-month rosuvastatin treatment in median plasma concentrations of D-dimer, IL-8, 
Table 1. Demographic, Clinical, and Laboratory Characteristics of Patients Who Completed the Study and Were Analyzed

\begin{tabular}{|c|c|}
\hline Total number & 54 \\
\hline Males, no. (\%) & $45(83.3)$ \\
\hline White subjects, no. (\%) & $51(94.4)$ \\
\hline Age (years), median (IQR) & $46.8(40.6-55.9)$ \\
\hline \multicolumn{2}{|l|}{ HIV transmission risk category, no. (\%) } \\
\hline IDU & $3(5.5)$ \\
\hline MSM & $28(51.8)$ \\
\hline Heterosexual & $23(42.6)$ \\
\hline $\begin{array}{l}\text { Nadir CD4 }{ }^{+} \text {lymphocyte count } \\
\quad\left(\text { cells } / \mathrm{mm}^{3}\right), \text { median (IQR) }\end{array}$ & $223(149-315)$ \\
\hline $\begin{array}{l}\text { Current } \mathrm{CD}^{+} \text {lymphocyte count } \\
\left(\text { cells } / \mathrm{mm}^{3}\right), \text { median (IQR) }\end{array}$ & $564(388-767)$ \\
\hline $\begin{array}{l}\text { Duration of HIV infection (years), } \\
\text { median (IQR) }\end{array}$ & $9.7(4.8-14.7)$ \\
\hline $\begin{array}{l}\text { Duration of current cART (months), } \\
\text { median (IQR) }\end{array}$ & $37(25-49)$ \\
\hline $\begin{array}{l}\text { Cumulative exposure to cART (years), } \\
\text { median (IQR) }\end{array}$ & $7.6(5.5-13.7)$ \\
\hline $\begin{array}{l}\text { Duration of plasma HIV RNA } \\
<20 \text { copies/ml with current cART } \\
\text { (months), median (IQR) }\end{array}$ & $34(23-46)$ \\
\hline BMI $\left(\mathrm{Kg} / \mathrm{m}^{2}\right)$, median (IQR) & $23.2(21.9-24.8)$ \\
\hline Cigarette smokers, no. (\%) & $31(57.4)$ \\
\hline $\begin{array}{l}\text { Patients with arterial hypertension, } \\
\text { no. }(\%)\end{array}$ & $9(16.7)$ \\
\hline Glucose (mg/dl), median (IQR) & $82(61-107)$ \\
\hline Insulin (ng/mol), median (IQR) & $0.51(0.32-0.84)$ \\
\hline Total cholesterol (mg/dl), median (IQR) & $239(186-262)$ \\
\hline LDL cholesterol (mg/dl), median (IQR) & $141(119-172)$ \\
\hline HDL cholesterol (mg/dl), median (IQR) & $49(35-64)$ \\
\hline Triglycerides (mg/dl), median (IQR) & $256(211-295)$ \\
\hline $\begin{array}{l}\text { Patients with total cholesterol } \\
>200 \mathrm{mg} / \mathrm{dl} \text {, no. }(\%)\end{array}$ & $47(87)$ \\
\hline $\begin{array}{l}\text { Patients with triglycerides }>200 \mathrm{mg} / \mathrm{dl} \text {, } \\
\text { no. }(\%)\end{array}$ & $38(70.4)$ \\
\hline \multicolumn{2}{|l|}{ Current cART, no (\%) } \\
\hline Tenofovir/emtricitabine & $32(59.2)$ \\
\hline Abacavir/lamivudine & $22(40.7)$ \\
\hline Efavirenz & $24(44.4)$ \\
\hline Darunavir/ritonavir & $18(33.3)$ \\
\hline Atazanavir/ritonavir & $12(22.3)$ \\
\hline
\end{tabular}

IQR, interquartile range; IDU, injection drug users; MSM, men who have sex with men; cART, combination antiretroviral therapy; BMI, body mass index; LDL, low-density lipoprotein; HDL, highdensity lipoprotein.

IL-10, and IL-12 in the whole study population. A statistically significant decrease in median plasma concentration of D-dimer in comparison with the baseline value was reported after both 6 months $(-20.2 \%)$ and 12 months $(-21.4 \%)$. With regard to the inflammation markers, a significant reduction in median plasma levels both at month 6 and at month 12 was observed for IL-8 (-21.7\% and $-24.6 \%$, respectively) and IL$12(-23.4 \%$ and $-18.7 \%$, respectively). On the contrary, median concentration of IL-10 decreased but not significantly during the 12-month follow-up.

Changes in coagulation and inflammation markers were also analyzed according to the current cART. When considering the subgroups of patients taking tenofovir/emtricitabine (32 subjects) or abacavir/lamivudine (22 subjects), we found

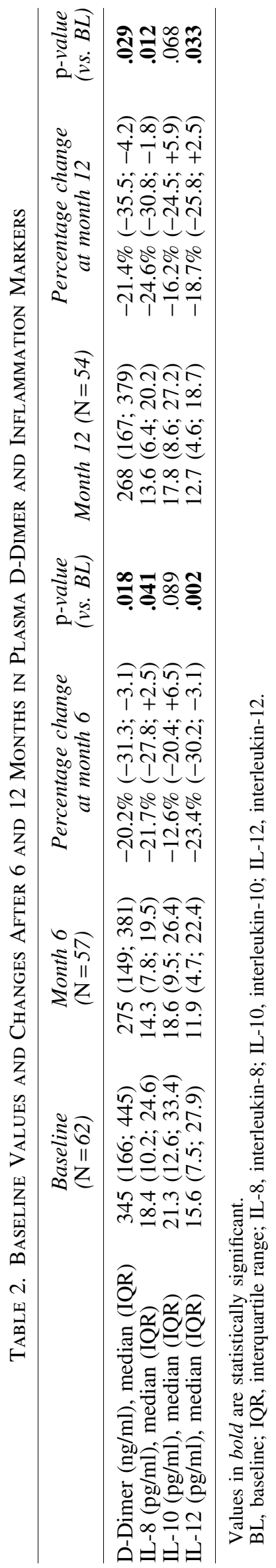


that changes in D-dimer, IL-8, and IL-12 were significant between baseline and month 12 for each subgroup, without significant difference between these two subgroups (Table 3 ). In the same way, changes between baseline and month 12 in D-dimer, IL-8, and IL-12 were significant in both subgroups of patients taking a PI/r (30 subjects) or efavirenz (24 subjects), without significant difference between these two subgroups (Table 4). On the contrary, changes from baseline to month 12 in serum concentration of IL-10 were not significant in all these four subgroups (Tables 3 and 4).

After 12 months of treatment, rosuvastatin led to a statistically significant reduction in median levels of total cholesterol, LDL cholesterol, and triglycerides versus respective baseline values. The observed changes in median lipid concentrations were $-23.2 \%$ (IQR, $-30.3 ;-9.8 ; p<.001$ ) for total cholesterol, $-25.4 \%$ (IQR, $-32.1 ;-10.6 ; p=.021$ ) for LDL cholesterol, and $-19.2 \%$ (IQR, $-27.8 ;-5.6 ; p=.027$ ) for triglycerides. The change in median HDL cholesterol was not significant $(-4.2 \%$; IQR, $-9.4 ;+3.3 ; p=.766)$.

Changes in median levels of glucose and insulin were not significant. Median glucose concentration (IQR) at baseline and after 12 months was $82(61-107)$ and $84(63-106) \mathrm{mg} / \mathrm{dl}$, respectively ( $p=.388)$; median insulin concentration (IQR) at baseline and after 12 months was $0.51(0.32-0.84)$ and 0.58 $(0.41-0.89) \mathrm{ng} / \mathrm{mol}$, respectively $(p=.078)$.

When considering immunological and virological parameters, mean $\mathrm{CD}^{+}$lymphocyte count and percentage of patients with undetectable plasma HIV viral load did not significantly change during the hypolipidemic treatment, showing neither appreciable interaction between antiretroviral drugs and rosuvastatin nor significant immunological effects of the lipidlowering agents. Change in median $\mathrm{CD}^{+}$lymphocyte count during the 12-month follow-up was +41 cells $/ \mathrm{mm}^{3}$ (IQR, -12 ; $+79 ; p=.578$ ) and all the 54 evaluated patients had plasma HIV RNA $<20$ copies $/ \mathrm{ml}$ at month 12 . A virological failure was observed in 3 out of the 62 enrolled patients (4.8\%), leading to a change in current cART, but genotype testing did not show any resistance mutations for the antiretroviral drugs. The adherence level to cART was $<95 \%$ in all these three patients. A viral blip was observed in five subjects $(8.1 \%)$, but no viral blips were reported in patients with virological failure.

Other parameters, which could produce some effects on the inflammation biomarkers, did not present significant variations during the entire follow-up period. The median BMI (IQR) changed from $23.2(21.9-24.8) \mathrm{kg} / \mathrm{m}^{2}$ at baseline to $23.1(21.7-24.9) \mathrm{kg} / \mathrm{m}^{2}$ at month $12(p=.702)$. Similarly, the number of cigarette smokers changed from $31(57.4 \%)$ to $30(55.5 \% ; p=.887)$.

Serious toxicity associated with discontinuing statin therapy occurred in 4 out of the 62 enrolled patients (6.4\%) and was represented by diarrhea in 2 cases and nausea in 2 cases. Toxicity not associated with statin discontinuation occurred in 13 patients (20.9\%) and was represented by gastrointestinal symptoms (nausea and diarrhea) in 8 subjects (12.9\%), myalgias in $5(8.1 \%)$, headache in $4(6.4 \%)$, and mild elevation in the creatine kinase (CK) concentration (always below $300 \mathrm{U} / \mathrm{L})$ in $4(6.4 \%)$.

\section{Discussion}

Several clinical studies have proved the anti-inflammatory effects of statins and their ability to reduce the serum

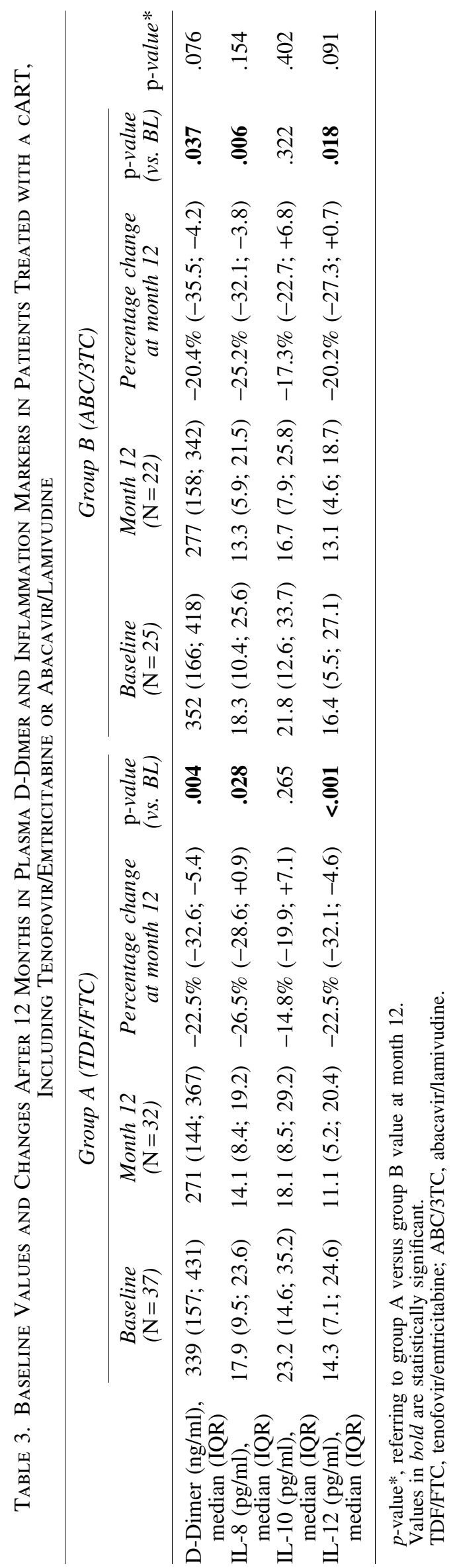




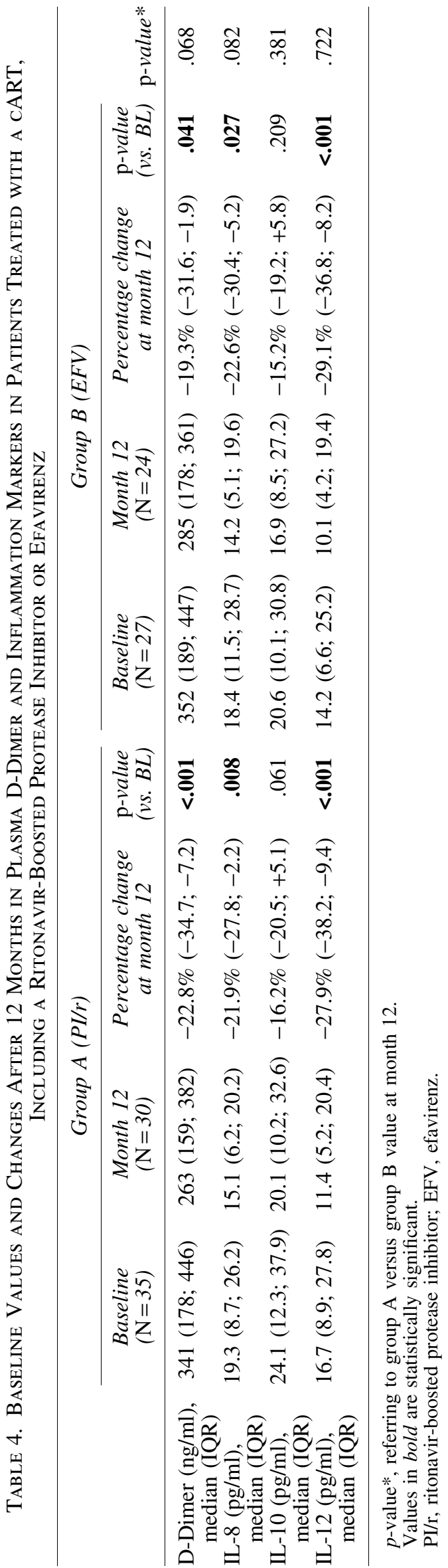

concentration of some inflammatory biomarkers among HIVinfected patients.

A randomized, double-blind trial investigated the effect of a 45-day course of pravastatin (40 mg daily) or rosuvastatin (10 mg daily) on the high-sensitive $\mathrm{C}$-reactive protein (hsCRP) concentration in 58 dyslipidemic HIV-positive patients. All the enrolled patients were treated with a PI/r-based cART and had a baseline hsCRP $<10 \mathrm{mg} / \mathrm{L}$. At the end of follow-up, statins led to a significant decrease in the median hsCRP level ( $-20 \%$ overall), without correlation with changes in lipid parameters. No significant reductions were reported in median levels of other biomarkers, such as soluble tumor necrosis factor- $\alpha$ receptor- 1 and -2 (TNFR-1 and -2), intercellular adhesion molecule-1 (ICAM-1), and vascular cell adhesion molecule-1 (VCAM-1). ${ }^{14}$

In a previous retrospective analysis of $151 \mathrm{HIV}$-infected patients receiving a stable cART including one PI/r and affected by hypercholesterolemia, we have assessed the antiinflammatory effect of treatment with rosuvastatin $(10 \mathrm{mg}$ daily), atorvastatin (10 mg daily), or pravastatin (40 mg daily). After 12 months, all statins led to a similar and significant decrease in serum concentration of hsCRP $(-23 \%$ overall $)$ and tumor necrosis factor- $\alpha$ (TNF- $\alpha)(-28 \%$ overall), without correlation between inflammation markers and lipid parameters. ${ }^{15}$

We have also evaluated the effects of rosuvastatin plus cART on several biomarkers (hsCRP, IL-6, IL-8, and TNF- $\alpha$ ) in a prospective, observational study, including 86 antiretroviral-naive HIV-infected patients starting tenofovir/ emtricitabine/efavirenz. After 12 months, patients with hypercholesterolemia and treated with cART plus rosuvastatin (10 mg daily) had a significantly greater decrease in serum levels of hsCRP and TNF- $\alpha$ than those with normal cholesterol levels and taking cART only. Changes in hsCRP were $-35.1 \%$ versus $-8.2 \%$, and changes in TNF- $\alpha$ were $-22.4 \%$ versus $-5.4 \%$, respectively. ${ }^{16}$

In a randomized controlled trial, $50 \mathrm{HIV}$-positive persons with mixed dyslipidemia were treated for 3 months with high-dose rosuvastatin $(40 \mathrm{mg}$ daily) or $10 \mathrm{mg} /$ day rosuvastatin plus $2 \mathrm{~g}$ /day of omega-3 polyunsaturated fatty acids (PUFAs) to assess changes in plasma levels of vascular endothelial growth factor (VEGF) and IL-8. Both rosuvastatin monotherapy and rosuvastatin plus PUFAs reduced significantly the IL-8 levels, while only high-dose rosuvastatin reduced significantly the VEGF values. ${ }^{17}$

The SATURN randomized, placebo-controlled trial evaluated the effects of rosuvastatin $(10 \mathrm{mg}$ daily) on the inflammation and immune activation markers in $147 \mathrm{HIV}-$ infected patients under cART, with normal LDL cholesterol, but with evidence of either heightened $\mathrm{T}$ cell activation or level of high-sensitivity C-reactive protein (hsCRP) $\geq 2 \mathrm{mg} / \mathrm{L}$. After 48 weeks, rosuvastatin led to a significant reduction in soluble CD14 (sCD14), lipoprotein-associated phospholipase A2, and interferon gamma-inducible protein-10 (IP-10) concentrations compared to placebo. ${ }^{18}$

Data about the immunomodulatory effects of statins are more limited and conflicting, in part.

A randomized, double-blind, placebo-controlled study investigated the effect of high-dose atorvastatin ( $80 \mathrm{mg}$ daily) on HIV RNA and cellular markers of immune activation in 24 HIV-infected subjects not receiving cART. After 8 weeks of therapy, atorvastatin did not affect plasma HIV RNA but significantly decreased the proportion of activated $\mathrm{T}$ 


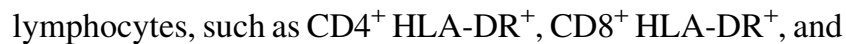
$\mathrm{CD}^{+}$HLA-DR $^{+} \mathrm{CD}^{+} 8^{+}$T cells. $^{19}$

In the SATURN trial, a 24-week treatment with rosuvastatin led to a significant decrease in serum markers of monocyte activation, such as sCD14 and tissue factorpositive patrolling monocytes, but no changes in levels of $\mathrm{T}$ cell activation were observed. ${ }^{20}$ In a randomized, placebocontrolled study including 28 treatment-naive HIV-infected men, rosuvastatin ( $20 \mathrm{mg}$ daily) for 8 weeks produced a small but significant increase in the CD4/CD8 T lymphocyte ratio, but did not influence other markers of $\mathrm{T}$ cell activation. ${ }^{21}$

The IMEA 043-CESAR study is a pilot prospective study investigating changes in the proportion of activated $\mathrm{T}$ lymphocytes in $43 \mathrm{HIV}$-infected subjects under suppressive cART, with CD4 count $<500$ cells $/ \mathrm{mm}^{3}$ and treated with rosuvastatin ( $20 \mathrm{mg}$ daily) for 12 weeks. At the end of statin treatment, the proportion of $\mathrm{CD}^{+} \mathrm{T}$ lymphocytes coexpressing CD38 and HLA-DR did not change significantly, but the proportion of $\mathrm{CD} 38^{+} \mathrm{CD} 8 \mathrm{~T}$ cells significantly decreased and this reduction remained significant 12 weeks after the statin interruption. Particularly, the proportion of activated CD8 T lymphocytes significantly decreased only in the subgroup of subjects with high baseline CD8 T cell activation. No significant variations in the plasma levels of hsCRP, IL-6, CD14, and D-dimers between baseline and week 12 were reported. ${ }^{22}$

In our observational analysis, a 12-month treatment with rosuvastatin at $10 \mathrm{mg}$ daily was effective to significantly reduce plasma levels of D-dimer and of two inflammatory markers (IL-8 and IL-12) in 54 HIV-infected patients under suppressive cART. This is the second study investigating the effect of statins on the D-dimer among HIV-positive persons, after the study by Weiss et $a l .{ }^{22}$ but is the first study showing a significant decrease in the D-dimer level associated with statin therapy in HIV-positive people.

IL-8 is a chemokine produced by macrophages and endothelial cells, which induces chemotaxis in neutrophils and angiogenesis. IL-12 is an interleukin produced by macrophages and dendritic cells, which is involved in the differentiation of naive T cells into Th1 cells. These chemokines are key mediators associated with systemic inflammation and with endothelial dysfunction, ${ }^{23}$ but no data about these markers in HIV-infected patients on statin therapy were published to date. So, in the present study, we have evaluated changes in D-dimer, IL-8, IL-10, and IL-12 to analyze the effect of statin treatment on these coagulation and inflammation markers, while its effect on hsCRP, IL-6, and TNFalpha was already evaluated in our previous reports. ${ }^{15,16}$

Moreover, the observed changes in serum levels of Ddimer and biomarkers, even if statistically significant, are quite small, so their clinical significance is still unknown, in particular, in patients with a low cardiovascular disease risk. However, the capability of rosuvastatin in decreasing plasma levels of these biomarkers seems to confirm the potential role of these lipid-lowering drugs as anti-inflammatory agents among HIV-infected subjects.

Although some authors have reported an increased risk of insulin resistance and diabetes mellitus in association with statins among HIV-infected patients, ${ }^{24,25}$ in the present study, rosuvastatin treatment did not alter serum levels of both glucose and insulin, in conformity with our previous analyses. ${ }^{26}$

Obviously, there are several limitations to our study, including the observational, noncontrolled design and the small number of enrolled patients. Moreover, the short observation period may have limited the accuracy of the analysis. Many patients with high cardiovascular disease risk (known cardiovascular disease or currently treated with beta-blockers or thiazidic diuretics) were excluded from the study, so the final analysis did not include a group of subjects who could take great advantage from the statin effects. Finally, the concomitant antiretroviral therapy is not the same, and this difference could certainly influence the anti-inflammatory effects of statin.

In conclusion, the anti-inflammatory properties of statins in HIV-positive patients are emerging with growing evidence, but larger randomized studies are certainly needed to better define their potential role as anti-inflammatory agents in this population.

\section{Author Disclosure Statement}

The authors have no conflicts of interest to declare. No funding was received for this study.

\section{References}

1. Schouten J, Wit FW, Stolte IG, Kootstra Na, et al.: Crosssectional comparison of the prevalence of age-associated comorbidities and their risk factors between HIV-infected and uninfected individuals: The AGEhIV cohort study. Clin Infect Dis 2014;59:1787-1797.

2. Guaraldi G, Prakash M, Moecklinghoff C, Stellbrink HJ: Morbidity in older HIV-infected patients: Impact of longterm antiretroviral use. AIDS Rev 2014;16:75-89.

3. Deeks SG, Lewin SR, Havlir DV: The end of AIDS: HIV infection as a chronic disease. Lancet 2013;382:1525-1533.

4. Sodora DL, Silvestri G: Immune activation and AIDS pathogenesis. AIDS 2008;22: 439-446.

5. Ridker PM, Danielson E, Fonseca FA, et al.: Reduction in C-reactive protein and LDL-cholesterol and cardiovascular event rates after initiation of rosuvastatin: A prospective study of the JUPITER trial. Lancet 2009;373:1175-1182.

6. Albert MA, Danielson E, Rifai N, Ridker PM: Effect of statin therapy on C-reactive protein levels: The Pravastatin Inflammation/CRP Evaluation (PRINCE): A randomized trial and cohort study. JAMA 2001;286:64-70.

7. Jain MK, Ridker PM: Anti-inflammatory effects of statins: Clinical evidence and basic mechanisms. Nat Rev Drug Discov 2005;4:977-987.

8. Kostapanos MS, Rizos CV, Elisaf MS: Benefit-risk assessment of rosuvastatin in the treatment of atherosclerosis and related diseases. Drug Saf 2014;37:481-500.

9. Phillip Owens III A, Mackman N: The antithrombotic effects of statins. Ann Rev Med 2014;65:433-445.

10. Antoniades C, Channon KM: Statins: Pleiotropic regulators of cardiovascular redox state. Antioxid Redox Signal 2014; 20:1195-1197.

11. Sahebkar A, Serban C, Mikhailidis DP, et al.: Association between statin use and plasma D-dimer levels. A systematic review and meta-analysis of randomised controlled trials. Thromb Haemost 2015;114:546-557.

12. Eckard AR, McComsey GA: The role of statins in the setting of HIV infection. Curr HIV/AIDS Rep 2015;12: 305-312.

13. Carr A: Statins as anti-inflammatory therapy in HIV disease? J Infect Dis 2011;203:751-752.

14. Aslangul E, Fellahi S, Assoumou LK, Bastard JP, Capeau J, Costagliola D. High-sensitivity C-reactive protein fall during 
statin therapy in HIV-infected patients receiving ritonavirboosted protease inhibitors. AIDS 2011;25:1128-1131.

15. Calza L, Trapani F, Bartoletti M, et al.: Statin therapy decreases serum levels of high-sensitivity C-reactive protein and tumor necrosis factor-alpha in HIV-infected patients treated with ritonavir-boosted protease inhibitors. HIV Clin Trials 2012;13:153-161.

16. Calza L, Vanino E, Salvadori C, et al.: Tenofovir/ emtricitabine/efavirenz plus rosuvastatin decrease serum levels of inflammatory markers more than antiretroviral drugs alone in antiretroviral-naive HIV-infected patients. HIV Clin Trials 2014;15:1-13.

17. Chantzichristos VG, Agouridis AP, Moutzouri E, Stellos K, Elisaf MS, Tselepis AD: Plasma VEGF and IL-8 levels in patients with mixed dyslipidemia. Effect of rosuvastatin monotherapy or its combination at a lower dose with omega3 fatty acids: A pilot study. Curr Vasc Pharmacol 2016 [Epub ahead of print]. PMID: 27041244.

18. Funderburg NT, Jiang Y, Debanne SM, et al:: Rosuvastatin reduces vascular inflammation and $\mathrm{T}$-cell and monocyte activation in HIV-infected subjects on antiretroviral therapy. J Acquir Immune Defic Syndr 2015;68:396-404.

19. Ganesan A, Crum-Cianflone N, Higgins J, et al.: High dose atorvastatin decreases cellular markers of immune activation without affecting HIV-1 RNA levels: Results of a double-blind randomized controlled clinical trial. J Infect Dis 2011;203:756-764.

20. Funderburg NT, Jiang Y, Debanne SM, et al:: Rosuvastatin treatment reduces markers of monocyte activation in HIVinfected subjects on antiretroviral therapy. Clin Infect Dis 2014;58:588-595.

21. Weijma RG, Vos ER, Ten Oever J, et al.: The effect of rosuvastatin on markers of immune activation in treatment- naïve human immunodeficiency virus-patients. Open Forum Infect Dis 2015;3:ofv201.

22. Weiss L, Chevalier MF, Assoumou L, et al.: Rosuvastatin is effective to decrease CD8 T-cell activation only in HIVinfected patients with high residual T-cell activation under antiretroviral therapy. J Acquir Immune Defic Syndr 2016; 71:390-398.

23. Vignali DA, Kuchroo VK: IL-12 family cytokines: Immunological playmakers. Nat Immunol 2012;13:722-728.

24. Erlandson KM, Jiang Y, Debanne SM, McComsey GA: Rosuvastatin worsens insulin resistance in HIV-infected adults on antiretroviral therapy. Clin Infect Dis 2015;61: 1566-1572.

25. Lichtenstein KA, Hart RL, Wood KC, et al.: Statin use is associated with incident diabetes mellitus among patients in the HIV Outpatient Study. J Acquir Immune Defic Syndr 2015;69:306-311.

26. Calza L, Colangeli V, Magistrelli E, et al.: No correlation between statin exposure and incident diabetes mellitus in HIV-1-infected patients receiving combination antiretroviral therapy. HIV Med 2016;17:631-633.

Address correspondence to: Leonardo Calza Department of Medical and Surgical Sciences Clinics of Infectious Diseases “Alma Mater Studiorum” University of Bologna S. Orsola-Malpighi Hospital via G. Massarenti 11 Bologna I-40138 Italy

E-mail: leonardo.calza@unibo.it 